\title{
First Galactic Survey of Energy-Dependent Diffusion by HAWC
}

\author{
Chad Brisbois* \\ Affiliation: Michigan Technological University, Houghton, Michigan, USA \\ E-mail: cbrisboi@mtu.edu
}

Hao Zhou

Affiliation: Los Alamos National Laboratory, Los Alamos, New Mexico, USA

\section{For the HAWC Collaboration}

Full author list and acknowledgements:

https: / / www . hawc-observatory . org/collaboration/icrc2019.php

See PoS(ICRC2019)1177

\begin{abstract}
HAWC's wide field-of-view enables unbiased observations of much of the galaxy, allowing for analysis of many candidate sources in the HAWC dataset. Recent work has allowed HAWC to start investigating electron diffusion around pulsars at $\mathrm{TeV}$ energies. Surveying electron diffusion at $\mathrm{TeV}$ energies along the galactic plane is a unique capability of HAWC that allows us to examine the sources of electrons in our galaxy and constrain the energy dependence of the diffusion mechanism. HAWC has already applied this model to the Geminga and Monogem pulsars. This work will expand the study to include multiple sources associated with pulsars in the latest HAWC dataset. Unlike older pulsars, lepton transport around young pulsars is likely to be dominated by advective processes. Using simple assumptions, both advective and diffusive processes predict that the size of the emission region follows a power law dependence on energy. This study has important consequences for the positron excess reported by space-based instruments; if electron diffusion is suppressed for tens of parsecs around pulsars relative to diffusion in the interstellar medium (a "two-zone" model), they may be able to explain at least part of the observed increase in the positron fraction above $10 \mathrm{GeV}$.
\end{abstract}

36th International Cosmic Ray Conference -ICRC2019-

July 24th - August 1st, 2019

Madison, WI, U.S.A.

${ }^{*}$ Speaker. 


\section{Introduction}

The discovery of very extended regions of $\mathrm{TeV} \gamma$-ray emission around the Geminga (PSR J0633+1746) and Monogem (B0656+14) pulsars [1] represented the first two examples of a new class of sources that have since been called $\mathrm{TeV}$ Halos [2]. Based on a proposed list of candidate pulsars from [2], a probable halo associated with PSR B0540+23 has since been found [3] by HAWC. This source class is currently poorly understood, but it may represent a fundamentally new understanding of particle transport in our galaxy [4]. Recent theoretical work has shown that Alfvén waves generated by the electrons and positrons produced by a pulsar themselves may produce the dramatically suppressed diffusion constants relative to that measured at Earth [4, 5]. Understanding the sources and transport of electrons and positrons in the Galaxy is an important component of interpreting the positron excess observed by PAMELA and others [6, 7]. Recent AMS results [7] indicate the presence of a source component in positron spectrum cutting off above $\sim 800 \mathrm{GeV}$. Further study of the diffusion of electrons and positrons around pulsars are needed to understand their properties.

\section{Analysis Description}

This analysis used the dataset containing 1128 days of HAWC data. The pulsars considered were from [2] (Seen in Table 1) with the addition of PSR J0633+0632 [8]. Of these, only 5 were in relatively isolated regions in the sky. Sources are fit using with a power law spectral assumption. The analysis is performed in a two step process: first a Gaussian morphology seeded with the pulsar position is fit to identify the centroid of $\mathrm{TeV}$ emission, then using the morphological model from [1] with the position fixed to determine the diffusion radius.

For this study, sources were fit in three Regions of Interest (ROI) due to their large angular extents. The first ROI contained the Crab Nebula and PSR B0540+23. The second ROI contained PSR J0633+1746, PSR B0656+14, and PSR J0633+063. Both of these regions can be seen in Figure 1. The third ROI contains PSR J1740+1000, which is relatively isolated in the HAWC dataset, it can be seen in Figure 2.

This lets us begin to test the prediction given in [2], that the population of TeV Halos have similar efficiencies $(\eta)$ to Geminga (PSR J0633+1746). Population studies such as this lets us begin to characterize the population of TeV Halos, whose efficiencies may depend on other things, such as the age of the halo. Results of these studies will be presented on the poster. 


\begin{tabular}{ccccc}
\hline Name & $\begin{array}{c}\text { Period } \\
\mathrm{s}\end{array}$ & $\begin{array}{c}\text { Distance } \\
\mathrm{kpc}\end{array}$ & $\begin{array}{c}\text { Age } \\
10^{5} \mathrm{yr}\end{array}$ & $\begin{array}{c}\dot{E} \\
10^{34} \mathrm{erg} \mathrm{s}^{-1}\end{array}$ \\
\hline \hline B0540+23* & 0.245975 & 1.56 & 2.53 & 4.09 \\
B0656+14* & 0.384929 & 0.29 & 1.11 & 3.80 \\
$\mathrm{~J}^{2} 633+063^{*}$ & 0.297395 & 1.35 & 0.592 & 12 \\
$\mathrm{~J} 1740+1000^{*}$ & 0.154087 & 1.23 & 1.14 & 23.2 \\
$\mathrm{~J} 0633+1746 *$ & 0.237099 & 0.19 & 3.42 & 3.25 \\
$\mathrm{~B} 1822-09$ & 0.769006 & 0.30 & 2.32 & 0.456 \\
$\mathrm{~B} 1951+32$ & 0.039531 & 3.00 & 1.07 & 374 \\
$\mathrm{~B} 1830-08$ & 0.085284 & 4.50 & 1.47 & 58.4 \\
$\mathrm{~J} 2032+4127$ & 0.143246 & 1.33 & 2.01 & 15.2 \\
$\mathrm{~J} 1913+0904$ & 0.163246 & 3.00 & 1.47 & 16.0 \\
$\mathrm{~J} 1831-0952$ & 0.067267 & 3.68 & 1.28 & 108 \\
$\mathrm{~J} 1913+1011$ & 0.035909 & 4.61 & 1.69 & 287 \\
\hline
\end{tabular}

Table 1: Pulsars in this list are from [2,8]. A * indicates if it is included in this study, with pulsar parameters taken from ATNF Catalog Version 1.60

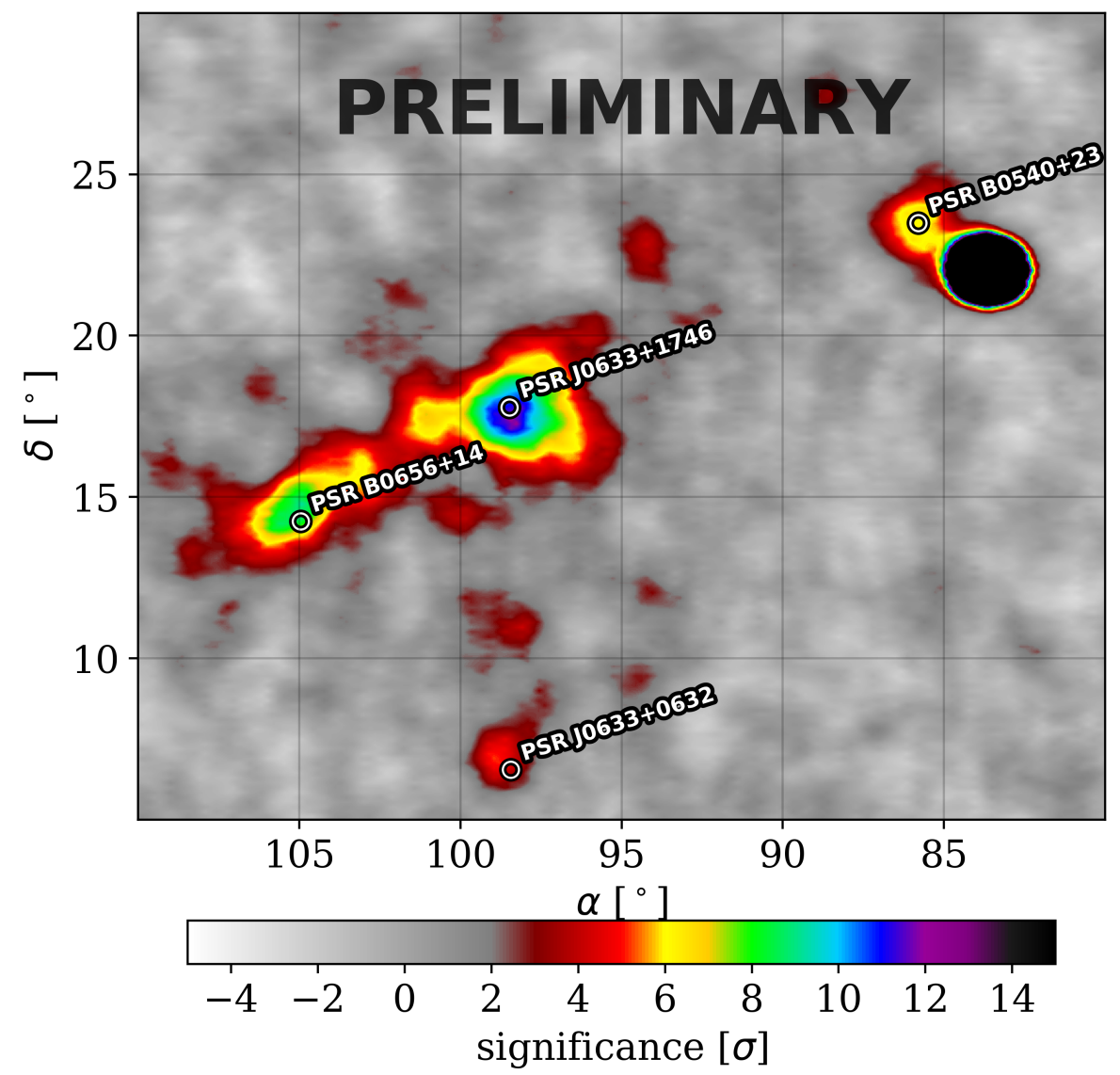

Figure 1: HAWC 1128 day significance maps assuming a -2.7 spectral index and $2^{\circ}$ smoothing. 


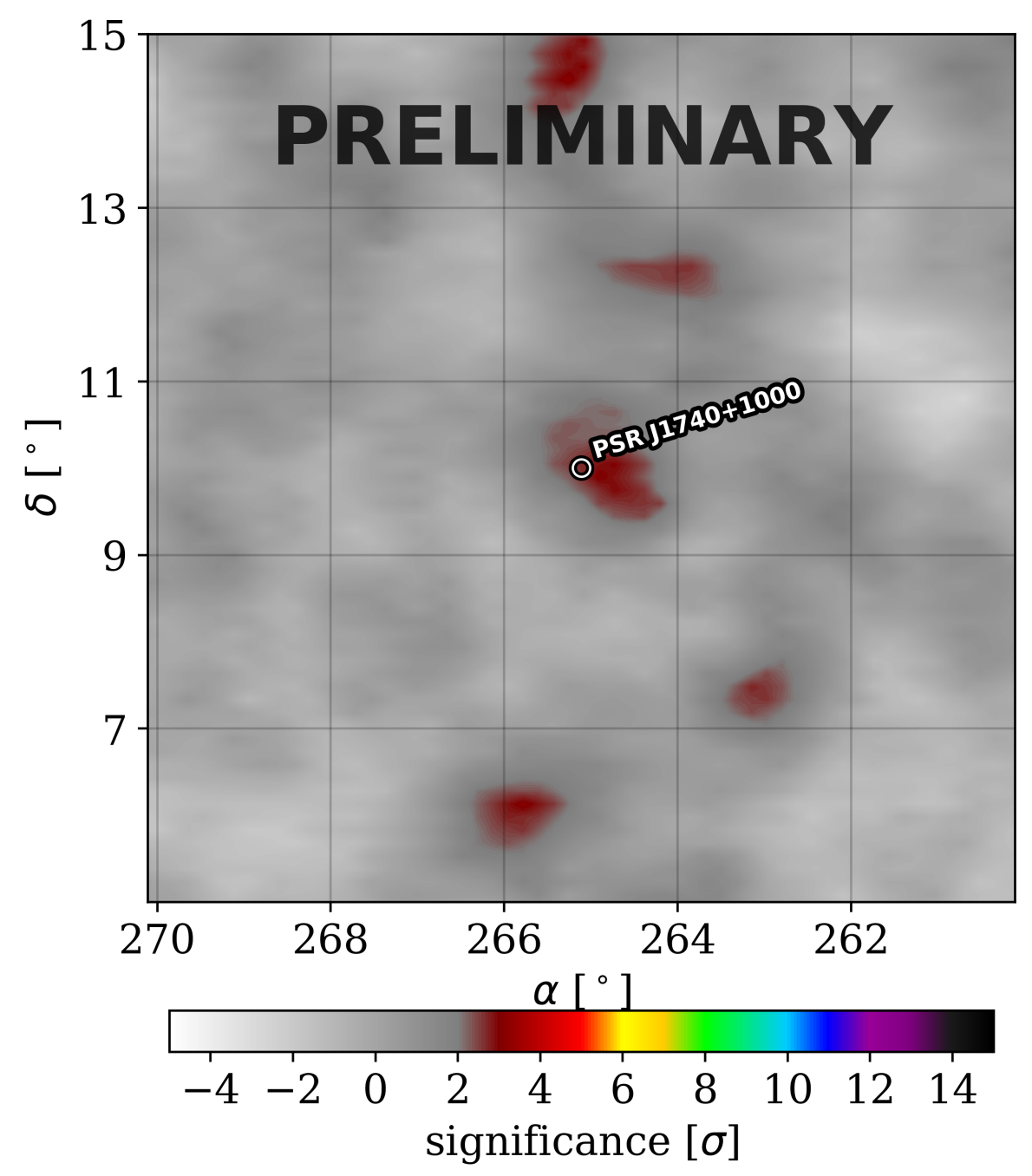

Figure 2: HAWC 1128 day significance maps assuming a -2.7 spectral index and $2^{\circ}$ smoothing. 


\section{References}

[1] A. U. Abeysekara, A. Albert, R. Alfaro, et al., Science 358 (2017) 911-914.

[2] T. Linden, K. Auchettl, J. Bramante, et al., Phys. Rev. D 96 (2017) 103016.

[3] C. Riviere, H. Fleischhack, and A. Sandoval, The Astronomer's Telegram 10941 (2017).

[4] C. Evoli, T. Linden, and G. Morlino, arXiv:1807.09263.

[5] D. Hooper and T. Linden, Phys. Rev. D 98 (2018) 083009.

[6] O. Adriani, G. C. Barbarino, G. A. Bazilevskaya, et al., Phys. Rev. Lett. 111 (2013) 081102.

[7] M. Aguilar, L. Ali Cavasonza, G. Ambrosi, et al., Phys. Rev. Lett. 122 (2019) 041102.

[8] C. Brisbois, C. Riviere, H. Fleischhack, and A. Smith, The Astronomer's Telegram 12013 (2018). 